\title{
Designing a warehouse internal layout using a parabolic aisles based method
}

\author{
Zhang, Z.Y. ${ }^{\mathrm{a},{ }^{*}}$, Liang, Y. ${ }^{\mathrm{b}}$, Hou, Y.P. ${ }^{\mathrm{a}}$, Wang, ${ }^{\mathrm{c}}{ }^{\mathrm{c}}$ \\ ${ }^{a}$ School of Logistics, Beijing Wuzi University, Beijing, P.R. China \\ ${ }^{b}$ Beijing Chaoyang District Committee of the Revolutionary Committee of the Chinese Kuomintang, Beijing, P.R. China \\ 'Manufacturing Engineering and Order Delivery Center, Beiqi Foton Motor Co., Ltd, Beijing, P.R. China
}

\begin{abstract}
A B S T R A C T
Refined layout is a basis of warehousing efficiency. Straight aisle is a typical feature of current warehouse internal layouts. The purpose of this paper is to explore the possibility of using curve aisles for warehouse layout. By Choosing typical non-traditional layouts and transforming their inclined cross-aisle trajectory into parabola, two parabolic aisle layouts, parabolic Flying- $V$ and parabolic Fishbone, are constructed. For unit-load warehouses, based on the morphological characteristic analysis and the parabolic types selection, the picking distance model and the cross-aisle length formula are presented. Interval Numerical Simulation Method (INSM) and Genetic Algorithms (GA) are adopted to solve the model respectively in order to verify the results. This research breaks through the realistic situation of straight aisle leading warehouse layout, and enriches the relevant layout theory. The calculation results of 100 warehouses with different sizes show that the picking distance of parabolic Flying-V could be reduced by $0.22-0.62 \%$ compared with the straight layout, and the theoretical possible improvement space has been compressed by $2.42-12.26 \%$. Its length of cross-aisle is shortened by $-0.03-3.10 \%$. The picking distance of parabolic Fishbone could be only reduced by 0.02-0.04\%. The theoretical possible improvement space has been compressed by 1.27 $1.83 \%$. But its length of cross-aisle will increase by $4.63-19.50 \%$ significantly. We believe that the layout of non-rectangular complex special-shaped warehouses based on curve trajectory aisles would become an important research topic. In addition, after some necessary modifications to the objectives and constraints, the proposed method in this paper may also be used for the arrangement of machines and devices in a workshop in principle.
\end{abstract}

\author{
ARTICLE INFO \\ Keywords: \\ Layout design; \\ Warehouse internal layout; \\ Parabolic aisle layout; \\ Layout efficiency; \\ Simulation; \\ Optimization; \\ Interval numerical simulation \\ method (INSM); \\ Genetic algorithms (GA) \\ *Corresponding author: \\ zyfzzy@263.net \\ (Zhang, Z.Y.) \\ Article history: \\ Received 8 April 2021 \\ Revised 26 April 2021 \\ Accepted 5 May 2021

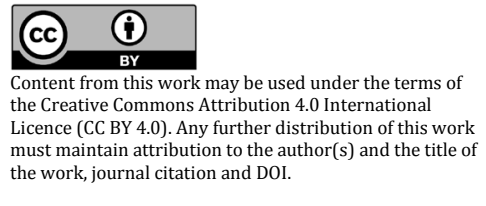

\section{References}

[1] Thomas, L.M., Meller, R.D. (2015). Developing design guidelines for a case-picking warehouse, International Journal of Production Economics, Vol. 170, Part C, 741-762, doi: 10.1016/i.ijpe.2015.02.011.

[2] Ardjmand, E., Shakeri, H., Singh, M., Sanei Bajgiran, O. (2018). Minimizing order picking makespan with multiple pickers in a wave picking warehouse, International Journal of Production Economics, Vol. 206, 169-183, doi: 10.1016/j.ijpe.2018.10.001.

[3] Yuan, T. (2016). Warehouse management, Third edition, Machinery Industry Press, Beijing, China, doi: cmpedu.com/books/book/2049485.htm.

[4] Gue, K.R., Meller, R.D. (2009). Aisle configurations for unit-load warehouses, IIE Transactions, Vol. 41, No. 3, 171182, doi: $10.1080 / 07408170802112726$.

[5] Pohl, L.M., Meller, R.D., Gue, K.R. (2010). Optimizing fishbone aisles for dual-command operations in a warehouse, Naval Research Logistics, Vol. 56, No. 5, 389-403, doi: 10.1002/nav.20355.

[6] Pohl, L.M., Meller, R.D., Gue, K.R. (2011). Turnover-based storage in non-traditional unit-load warehouse designs, IIE Transactions, Vol. 43, No. 10, 703-720, doi: 10.1080/0740817X.2010.549098. 
[7] Gue, K.R., Ivanović, G., Meller, R.D. (2012). A unit-load warehouse with multiple pickup and deposit points and non-traditional aisles, Transportation Research, Part E: Logistics and Transportation Review, Vol. 48, No. 4, 795806, doi: 10.1016/j.tre.2012.01.002.

[8] Cardona, L.F., Rivera, L., Martínez, H.J. (2012). Analytical study of the fishbone warehouse layout, International Journal of Logistics Research and Applications, Vol. 15, No. 6, 365-388, doi: 10.1080/13675567.2012.743981.

[9] Öztürkoğlu, Ö., Gue, K.R., Meller, R.D. (2012). Optimal unit-load warehouse designs for single-command operations, IIE Transactions, Vol. 44, No. 6, 459-475, doi: 10.1080/0740817X.2011.636793.

[10] Clark, K.A., Meller, R.D. (2013). Incorporating vertical travel into non-traditional cross aisles for unit-load warehouse designs, IIE Transactions, Vol. 45, No. 12, 1322-1331, doi: 10.1080/0740817X.2012.724188.

[11] Jiang, M.X., Feng, D.Z., Zhao, Y.L., Yu, M.F. (2013). Optimization of logistics warehouse layout based on the improved Fishbone layout, Systems Engineering - Theory \& Practice, Vol. 33, No. 11, 2920-2929, doi: sysengi.com/CN/Y2013/V33/I11/2920.

[12] Liu, Y.Q., Zhang, Y.H., Jiao, N. (2014). Slotting optimization allocation of storage based on fishbone, Logistics SciTech, Vol. 37, No. 12, 66-70.

[13] Öztürkoğlu, Ö., Gue, K.R., Meller, R.D. (2014). A constructive aisle design model for unit-load warehouses with multiple pickup and deposit points, European Journal of Operational Research, Vol. 236, No. 1, 382-394, doi: 10.1016/i.ejor.2013.12.023.

[14] Cardona, L.F., Soto, D.F., Rivera, L., Martínez, H.J. (2015). Detailed design of fishbone warehouse layouts with vertical travel, International Journal of Production Economics, Vol. 170, Part C, 825-837, doi: 10.1016/i.ijpe. 2015.03.006.

[15] Bortolini, M., Faccio, M., Gamberi, M., Manzini, R. (2015). Diagonal cross-aisles in unit load warehouses to increase handling performance, International Journal of Production Economics, Vol. 170, Part C, 838-849, doi: 10.1016/i.ijpe.2015.07.009.

[16] Liu, Q., Yang, P.H., Liu, R.Q., Yang, Y.Y. (2016). Optimization model of warehouse layout and determination of optimal angle based on genetic algorithms, Journal of Hebei North University (Natural Science Edition), Vol. 32, No. 3, 21-27, doi: j.issn.1673-1492.2016.03.006.

[17] Mesa, A. (2016). A methodology to incorporate multiple cross aisles in a non-traditional warehouse layout, Master's thesis, Ohio University, from http://rave.ohiolink.edu/etdc/view?acc num=ohiou1480669754531612, accessed June 1, 2021.

[18] Mowrey, C.H., Parikh, P.J., Gue, K.R. (2018). A model to optimize rack layout in a retail store, European Journal of Operational Research, Vol. 271, No. 3, 1100-1112, doi: 10.1016/j.ejor.2018.05.062.

[19] Zhang, Z.Y., Wang, Q., Liang, Y. (2019). Twin leaf method for warehouse internal layout and its aisles angle optimization, Systems Engineering, Vol. 37, No. 2, 70-80, from http://www.cnki.com.cn/Article/CJFDTotal-GCXT201 902007.htm, accessed June 1, 2021.

[20] Moder, J.J., Thornton, H.M. (1965). Quantitative analysis of the factors affecting floor space utilization of palletized storage, Journal of Industrial Engineering, Vol. 16, No. 1, 8-18.

[21] Francis, R.L. (1967). On some problems of rectangular warehouse design and layout, Journal of Industrial Engineering, Vol. 18, No. 10, 595-604.

[22] Francis, R.L. (1967). Sufficient conditions for some optimum-property facility designs, Operations Research, Vol. 15, No. 3, 448-466, doi: 10.1287/opre.15.3.448.

[23] Berry, J.R. (1968). Elements of warehouse layout, International Journal of Production Research, Vol. 7, No. 2, 105121, doi: $10.1080 / 00207546808929801$.

[24] White, J.A. (1972). Optimum design of warehouses having radial aisles, AIIE Transactions, Vol. 4, No. 4, 333-336, doi: $10.1080 / 05695557208974871$.

[25] Bassan, Y., Roll, Y., Rosenblatt, M.J. (1980). Internal layout design of a warehouse, AIIE Transactions, Vol. 12, No. 4, 317-322, doi: 10.1080/05695558008974523.

[26] Goldberg, D.E., Holland, J.H. (1988). Genetic algorithms and machine learning, Machine Learning, Vol. 3, 95-99, doi: 10.1023/A:1022602019183.

[27] Straka, M., Khouri, S., Lenort, R., Besta, P. (2020). Improvement of logistics in manufacturing system by the use of simulation modelling: A real industrial case study, Advances in Production Engineering \& Management, Vol. 15, No. 1, 18-30, doi: 10.14743/apem2020.1.346.

[28] Awaga, A.L., Xu, W., Liu, L., Zhang, Y. (2020). Evolutionary game of green manufacturing mode of enterprises under the influence of government reward and punishment, Advances in Production Engineering \& Management, Vol. 15, No. 4, 416-430, doi: 10.14743/apem2020.4.375.

[29] Sebo, J., Busa Jr., J. (2020). Comparison of advanced methods for picking path optimization: Case study of dualzone warehouse, International Journal of Simulation Modelling, Vol. 19, No. 3, 410-421, doi: 10.2507/IJSIMM193-521.

[30] Burinskiene, A., Lorenc, A., Lerher, T. (2018). A simulation study for the sustainability and reduction of waste in warehouse logistics, International Journal of Simulation Modelling, Vol. 17, No. 3, 485-497, doi: 10.2507/ IJSIMM17(3)446.

[31] Li, H.-Y., Xu, W., Cui, Y., Wang, Z., Xiao, M., Sun, Z.-X. (2020). Preventive maintenance decision model of urban transportation system equipment based on multi-control units, IEEE Access, Vol. 8, 15851-15869, doi: 10.1109/ ACCESS.2019.2961433. 


\title{
Oblikovanje notranje razporeditve skladišča z metodo paraboličnih prehodov
}

\author{
Zhang, Z.Y. ${ }^{\mathrm{a},{ }^{*}}$, Liang, Y. ${ }^{\mathrm{b}}$, Hou, Y.P. ${ }^{\mathrm{a}}$, Wang, Q. ${ }^{\mathrm{c}}$ \\ aschool of Logistics, Beijing Wuzi University, Beijing, P.R. China \\ bBeijing Chaoyang District Committee of the Revolutionary Committee of the Chinese Kuomintang, Beijing, P.R. China \\ 'Manufacturing Engineering and Order Delivery Center, Beiqi Foton Motor Co., Ltd, Beijing, P.R. China
}

\begin{abstract}
POVZETEK
Premišljena postavitev je osnova učinkovitosti skladiščenja. Ravni prehodi so tipična značilnost trenutnih notranjih razporeditev skladišč. Namen tega prispevka je raziskati možnost uporabe ukrivljenih prehodov za postavitev skladišča. Z izbiro tipičnih postavitev in s preoblikovanjem trajektorije ravnega prehoda v parabolo smo ustvarili dve parabolični postavitvi prehoda, parabolični leteči-V (angl. Flying-V) in parabolična ribja kost (angl. Fishbone). Za paletna skladišča sta na podlagi analize morfoloških značilnosti in izbire paraboličnih tipov predstavljena model razdalje do regala in formula za razdaljo med regali. Metoda intervalne numerične simulacije (INSM) in genetski algoritem (GA) sta uporabljena za rešitev modela in pridobitev rezultatov. Ta raziskava nadgradi realistično stanje postavitve skladišča $\mathrm{z}$ ravnimi prehodi in obogati obstoječo teorijo postavitve skladišč. Rezultati izračuna 100 skladišč z različnimi velikostmi kažejo, da bi se razdalja do regala v primeru postavitve skladišča po načelu paraboličnega letečega-V lahko zmanjšala za 0,22-0,62 \% v primerjavi z ravno postavitvijo, teoretični možni prostor za izboljšanje pa je bil stisnjen za 2,42-12,26 \%. Razdalja med regali se skrajša za -0,03-3,10 \%. Razdalja do regala $v$ primeru parabolične ribje kosti se lahko zmanjša le za 0,02-0,04 \%. Teoretični možni prostor za izboljšanje je bil skrčen za 1,27$1,83 \%$. Toda razdalja med regali se je znatno povečala za 4,63-19,50\%. Menimo, da bi postavitev nepravokotnih kompleksnih skladišč posebnih oblik na osnovi krivuljnih prehodov lahko postala pomembna raziskovalna tema. $\mathrm{Ob}$ vpeljavi potrebnih sprememb se predlagana metoda lahko uporabi tudi za razporeditev strojev in naprav v delavnici.
\end{abstract}

\section{PODATKI O ČLANKU}

Ključne besede:

Oblikovanje postavitve;

Notranja ureditev skladišča; Parabolična postavitev prehodov; Učinkovitost postavitve; Simulacija; Optimizacija;

Metoda intervalne numerične simulacije (INSM);

Genetski algoritmi (GA)

*Kontaktna oseba: zyfzzy@263.net (Zhang, Z.Y.)

Zgodovina članka: Prejet 8. aprila 2021 Popravljen 26. aprila 2021 Sprejet 5. maja 2021

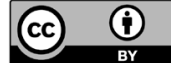

Content from this work may be used under the terms of the Creative Commons Attribution 4.0 International Licence (CC BY 4.0). Any further distribution of this work must maintain attribution to the author(s) and the title of the work, journal citation and DOI. 